\title{
The Weighted Support Vector Machine Based on Hybrid Swarm Intelligence Optimization for Icing Prediction of Transmission Line
}

\author{
Xiaomin Xu, Dongxiao Niu, Peng Wang, Yan Lu, and Huicong Xia \\ Research Institute of Technology Economics Forecasting and Assessment, School of Economics and Management, \\ North China Electric Power University, Beijing 102206, China \\ Correspondence should be addressed to Xiaomin Xu; xuxiaomin0701@126.com
}

Received 22 December 2014; Revised 14 March 2015; Accepted 14 March 2015

Academic Editor: Alejandro Ortega-Moñux

Copyright ( 2015 Xiaomin Xu et al. This is an open access article distributed under the Creative Commons Attribution License, which permits unrestricted use, distribution, and reproduction in any medium, provided the original work is properly cited.

\begin{abstract}
Not only can the icing coat on transmission line cause the electrical fault of gap discharge and icing flashover but also it will lead to the mechanical failure of tower, conductor, insulators, and others. It will bring great harm to the people's daily life and work. Thus, accurate prediction of ice thickness has important significance for power department to control the ice disaster effectively. Based on the analysis of standard support vector machine, this paper presents a weighted support vector machine regression model based on the similarity (WSVR). According to the different importance of samples, this paper introduces the weighted support vector machine and optimizes its parameters by hybrid swarm intelligence optimization algorithm with the particle swarm and ant colony (PSO-ACO), which improves the generalization ability of the model. In the case study, the actual data of ice thickness and climate in a certain area of Hunan province have been used to predict the icing thickness of the area, which verifies the validity and applicability of this proposed method. The predicted results show that the intelligent model proposed in this paper has higher precision and stronger generalization ability.
\end{abstract}

\section{Introduction}

Safe and reliable power supply is the security to ensure sound and rapid development of the national economy; as the main part of power transmission in the grid, normal and safe operation of transmission lines is an important guarantee for the grid to avoid a serious accident, while the icing on the transmission line will lead to excessive tension, conductor galloping, tripping, and break accident of transmission line. It will also cause the interruption of power supply, affecting the stability and security of power system operation seriously. Moreover, due to reverse distribution of China's resources and productivity, our country needs to vigorously promote construction of outgoing channel of power base. It will increase the possibility of the icing when transmission lines go through extremely harsh complex area of the contamination, high altitude, snow, strong acid rain, and fog $[1,2]$.

In the early 30 s of last century, Britain, Japan, Canada, and American had some reports on transmission line ice coating, which had caused safety accident and brought the huge economic loss [3]. As one of the serious transmission line icing countries, the probability of occurrence of ice disaster accident of transmission line in China stays the forefront in the world [4]. Icing of transmission lines has become one of the important factors affecting the safe operation of power grid in the world. In view of great harm to the power system operation brought by icing, in recent years, the related research work on conductor icing has gradually become a hot research at home and abroad. And they have made certain achievements in the aspect of the formation mechanism of transmission line icing, ice prevention measures, Icing image monitoring, and conductor icing prediction model. The methods used for the icing prediction include empirical models, statistical refinement model $[5,6]$, and the intelligent models, such as neural network [7-9], support vector machine $[10-13]$ and so on. The multivariate linear regression model is the most widely used in the statistical theory model. But the influence factors considered in the model are not 
comprehensive enough, and the model is required to meet the various statistical assumptions. There are restrictions on the types of influence factors, which limit the range of application [14]. Experience refined model is set out to establish a model from the physical essence of the ice cover and it is relatively simple; the generalization ability is weak. The outputs of different meteorological have high volatility [15]; neural network has the great ability of approaching nonlinearity but is easy to fall into local optimum and appear over learning situation. This model also has the disadvantages of low efficiency and poor generalization ability. It is difficult to guarantee the prediction accuracy of models [16]. As the climate factors have the characteristics of greater volatility and randomness, SVM can take comprehensive consideration of multiple factors of ice thickness and has better ability of nonlinear mapping and generalization [17]. And the model has the advantages of repeated training and the faster speed of convergence [18]; it can solve practical problems of the small sample, nonlinearity, local extreme value, and so on. It is widely used in many fields such as machine control and speech recognition and has achieved good forecasting effects [19].

The standard SVM has the same punishment for deviation and accuracy requirements for different samples. However, in practical applications, we often find that some samples have large correlation degree which requires a smaller training error, while some has small correlation degree which has the permissible of relatively large error [20]. At this point, the traditional SVM cannot obtain an accurate prediction result [21]. In view of this problem, given comprehensive consideration of the factors of the environmental temperature, relative humidity, wind speed, wind direction, and elevation effects, combined with statistical prediction model and icing meteorological parameters model used for icing prediction, this paper proposes a weighted support vector (WSVR) regression algorithm for icing prediction [22]. We determine the weights of different samples by calculating the correlation coefficient between samples and optimize the parameters $g$ and $C$ using hybrid optimization algorithm of particle swarm and ant colony (PSO-ACO) to improve the model's generalization ability. In the empirical analysis, ACOSVM, BP neural network and linear regression methods are used for comparison. By contrast, the model proposed in this paper has higher precision and can accurately predict the ice thickness. The establishment of the model has practical significance for power department to effectively control the ice disaster and improve the safe and reliable operation of power grid [23].

\section{Basic Theories}

\subsection{The Hybrid Cluster Intelligent Optimization}

2.1.1. Swarm Intelligence Optimization Algorithm. The basic theory of swarm intelligent optimization algorithm is to simulate exchanges and cooperation of the actual biological group lives between each individual, with a simple and limited individual behavior and intelligence to form the overall capability of the whole population of inestimable through interaction. Various organisms in a swarm intelligent optimization algorithm are handled by artificial, and individuals do not have the volume and quality of the actual biological. Its behavior has the necessary processing based on people's needs to solve the problem [24].

Swarm intelligence optimization algorithm is a search of probability essentially. It does not need gradient information of questions and has the following characteristics which are different from the traditional optimization algorithm.

(1) The interaction of individuals in the population is distributed, and there is no direct central control. Individual failure will not affect to solution of the problem. It has the strong robustness.

(2) Each individual can only perceive the local information and the individual's ability to follow the rules. So the swarm intelligence method is simple and convenient.

(3) The computing time is less and the platform is easy to expand.

(4) Self-organization, namely, the complex behavior of community is via a simple individual interaction which exhibits a high degree of intelligence.

Swarm intelligence optimization algorithm theory mainly aims to study the algorithm characteristics and improve the shortage and performance. The research mainly includes two aspects: one is to study its own characteristics of this algorithm to improve its performance; the other is to combine the swarm intelligence optimization with other algorithm to produce a new hybrid intelligent algorithm through the fusion of different algorithms.

\subsubsection{Hybrid Swarm Intelligence Optimization Algorithm.} Currently, the thinking of swarm intelligence is receiving increasing attention, which shows great characteristics in solving problems, especially optimization problems. There are many algorithms based on the group, such as genetic algorithm, differential evolution, ant colony optimization, particle swarm optimization, and evolutionary programming, which can be grouped into swarm intelligence algorithms. As the most commonly used swarm intelligence optimization algorithms, ant colony optimization and particle swarm optimization have greater optimization features. Ant colony optimization, which is the simulation of ant colony foraging process, has been successfully applied to many discrete optimization problems. Particle swarm optimization, which is the simulation of birds foraging process, is an efficient parallel search algorithm in continuous optimization field.

Ant colony optimization uses pheromone to transmit information, while particle swarm optimization uses three pieces of information of the information of its own, individual extreme information, and global extreme information to guide the particle to the next iteration. Using the organic combination of the positive feedback principle and some heuristic algorithms, ant colony optimization is easy to run into prematurity and fall into local optimum. The organic combination of those two algorithms can overcome the shortcomings of them effectively and improve the computational efficiency significantly. According to the mixing 
characteristics of ant colony optimization and particle swarm optimization, this paper proposes an improved ACO_PSO hybrid algorithm.

(1) Ant Colony Optimization. ACO is inspired by Italy scholar Dorigo $\mathrm{M}$ from the foraging behavior of real ant colony in nature. He found that an individual ant does not have much wisdom or master the nearby geographic information. But the colony can find an optimal path from nest to food sources. Established on the findings, Dorigo $M$ and other researchers proposed ACO theory in 1991, attracting research enthusiasm of many scholars. The basic ACO model consists of the following three equations:

$$
\begin{gathered}
P_{i j}^{k}=\frac{\tau_{i j}^{\alpha} \eta_{i j}^{\beta}}{\sum_{j \in \Lambda} \tau_{i j}^{\alpha} \eta_{i j}^{\beta}}, \\
\tau_{i j}(n+1)=\rho \tau_{i j}(n)+\sum_{k=1}^{m} \Delta \tau_{i j}^{k}, \\
\tau_{i j}^{k}=\frac{Q}{\sum L_{k}} .
\end{gathered}
$$

If the ant marked NO, $k$ passes by the path from $i$ to $j$, where $m$ is the number of ants, $n$ is the number of iterations, $i$ is the position of ants, $j$ is the position where ants can reach, $\Lambda$ is the set of the position where ants can reach, $\eta_{i j}$ is the heuristic information, which means the visibility of the path from $i$ to $j$, named $\eta_{i j}=1 / d_{i j}, L_{k}$ is the objective function, $\tau_{i j}$ is the pheromone intensity of the path from $i$ to $j, \tau_{i j}^{k}$ is the number of pheromone left by ants on the path from $i$ to $j$, $\alpha$ is the weight of the path, $\beta$ is the weight of the heuristic information, $\rho$ is the evaporation factor of the number of pheromone on the path, $Q$ is coefficient of the pheromone quality, and $P_{i j}^{k}$ denotes the transition probabilities of the NO $k$ ant moving from $i$ to $j$.

(2) Particle Swarm Optimization. Particle swarm optimization (PSO) is a kind of evolutionary algorithm, derived from the observation of the birds' behavior of searching for food. The conversion process of the motion of whole flock from disorderly to orderly comes from information shared by each individual bird in the flock, so as to find food [25].

The method used by PSO to solve optimization problems is to initialize a group of random particles and find the optimal solution through several iterations (Figure 1). In the process of iterations, each particle updates its direction and position constantly according to two extreme values. The first one is the optimal solution found by the particle itself, called individual extreme pbest, and the other one is the current optimal solution found by the entire particle swarm, named global extreme gbest. At the beginning of the iterations, the position of each initialized particle is the individual extreme, while the best position of the particle swarm is the global extreme. After all of the particles in the swarm complete the first iteration, we should compare the position front and rear of each particle and update the individual extreme with the optimal solution in this iteration if the new position is better

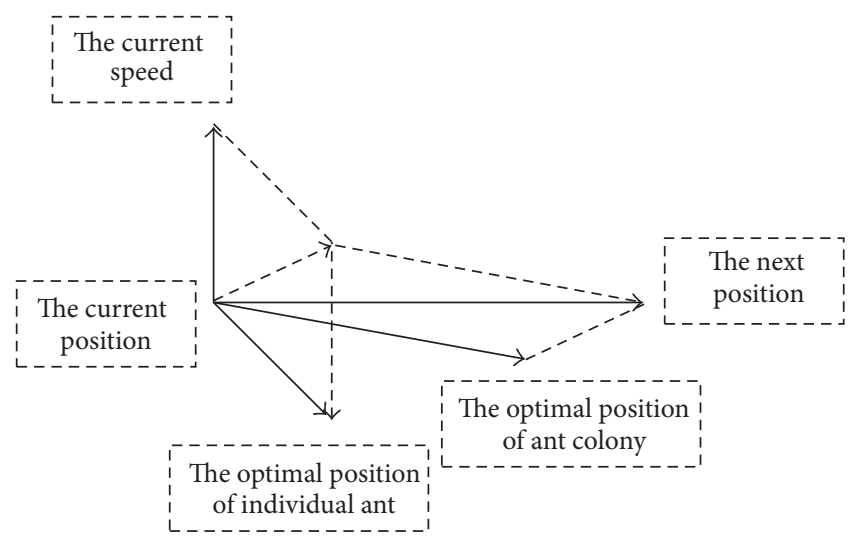

FIGURE 1: Movement of particles.

than the previous one. Then, we need to get the optimal solution throughout individual extremes of all particles in the swarm as global extreme by comparison and update the global extreme if the new one is better than the old one. The final global extreme obtained through these cycle iteration operations determines the optimal solution [26].

After obtaining individual extreme and global extreme in the process, each particle needs to update its velocity and position according to the following formulas:

$$
\begin{gathered}
v_{i, j+1}=w v_{i, j}+c_{1} * \operatorname{random}() *\left(\text { pbest }_{i, j}-P_{i, j}\right) \\
+c_{2} * \operatorname{random}() *\left(\text { gbest }_{i, j}-P_{i, j}\right), \\
P_{i, j+1}=P_{i, j}+v_{i, j+1},
\end{gathered}
$$

where $v_{i, j}$ denotes the velocity of the $i$ particle after $j$ iterations, $P_{i, j}$ means the position of the $i$ particle after $j$ iterations, pbest $t_{i, j}$ and $g$ best $t_{i, j}$ are on behalf of the individual extreme and global extreme of the $i$ particle after $j$ iterations, $w$ is the inertia weight of the updated speed to the speed of pre update, $\operatorname{random}()$ is a random number within $(0,1)$, and $c_{1}$ and $c_{2}$ are learning factors within $(0,2]$. The velocity of particles in the swarm is limited in $\left(0, v_{\max }\right)$, and the updated value should be replaced with $v_{\max }$ if it exceeds maximum $v_{\text {max }}$ in the process of iterations.

In the process of particle swarm optimization algorithm, particle shares global extreme value to other particles within the group. This one-way flow of shared information and data makes the whole search process follow the group within the current optimal solution. Therefore, the initial particle swarm optimization algorithm has fast global convergence capability.

2.1.3. ACO_PSO Hybrid Optimization Algorithm. Researches show that the initial search process of ant colony optimization operates slowly because of lack of initial pheromone, and the set continuous parameters $\alpha, \beta$, and $\rho$ are mainly determined by experimental methods, which makes it difficult to achieve the optimal value because of the relation of the accuracy, the calculation speed, the performance, and other aspects of the method to the experience of laboratory assistants. PSO 


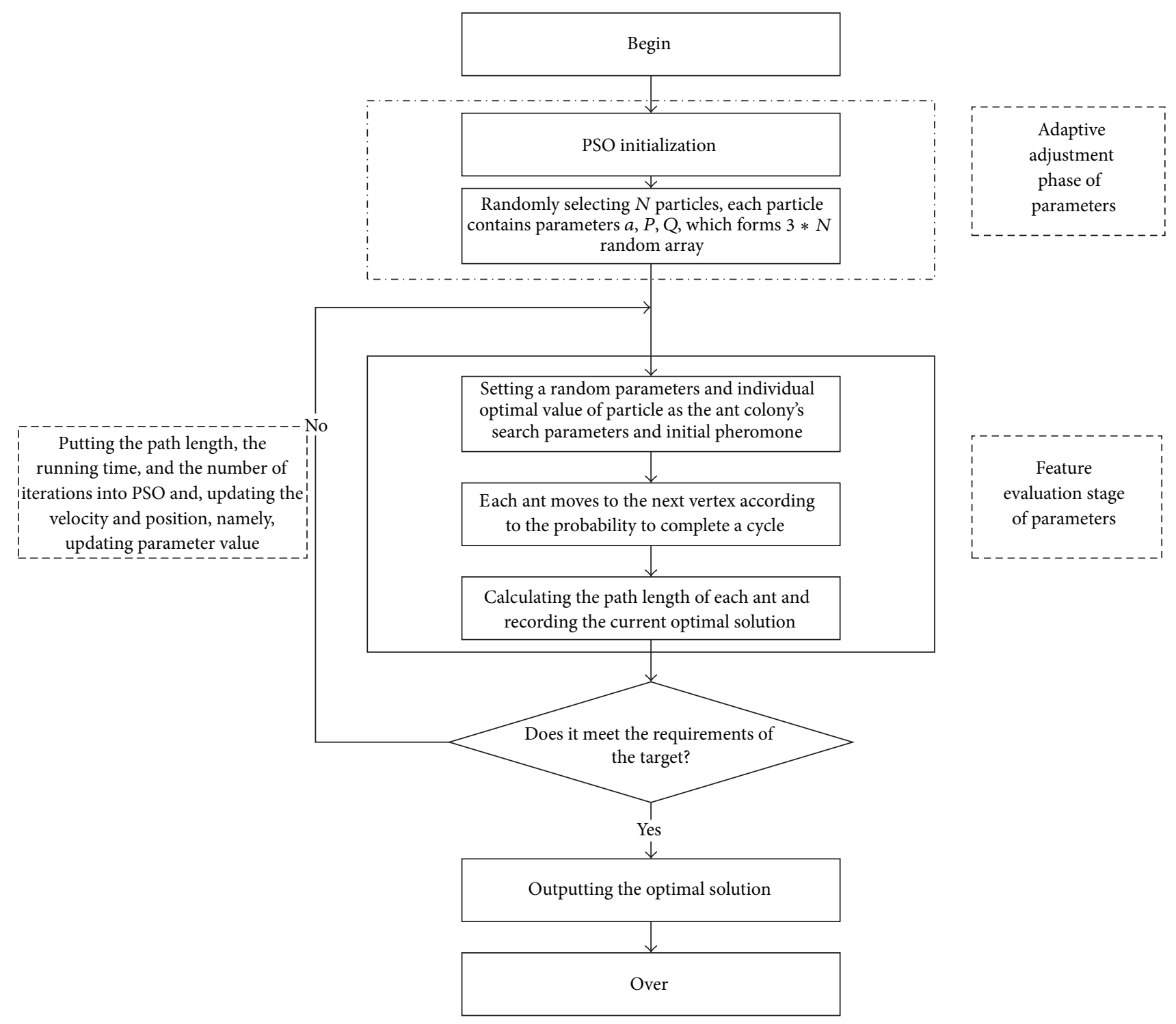

FIGURE 2: Flow chart of ACO-PSO hybrid intelligent optimization algorithm.

algorithm performs excellently in optimizing problems of continuous space, but when we get local optimal through $N$ iterations, the change in rate of particles is mainly affected by $w v_{i, j}$, while $w$ is in the range of 0 and 1 . That is to say, along with the increase in the number of iterations, the speed of the particles will become smaller and smaller, and tends to 0 gradually, which makes it easy to fall into local convergence. To overcome the disadvantages of the two algorithms, this paper will integrate and improve them and propose a hybrid algorithm based on particle swarm and ant colony. First, we use the particle swarm optimization algorithm for fast global search to determine the parameters of ant colony and transform the better value into initial information pheromone. Next, we use ant colony algorithm for path searching, at the same time we put the length of optimal solution, the running time, and the numbers of iterations calculated by this set of parameters into the PSO algorithm, and update the velocity and position of each particle according to formula until we get the optimal solution for ant colony algorithm $[27,28]$.

The basic steps of ACO-PSO hybrid algorithm are shown in Figure 2.

\subsection{The Weighted Support Vector Machine Algorithm}

2.2.1. Basic Theory of SVM. The principle of SVM is to transform the samples which cannot be separated in linear low-dimensional space to high dimensional feature space by nonlinear mapping algorithm to make the samples linearly separable and then analyze linearly the samples transformed. SVM regression is to create a nonlinear mapping and map the data to high dimensional feature space for the linear regression work later [21].

Assuming that the samples are $\left(x_{1}, y_{1}\right),\left(x_{2}, y_{2}\right), \ldots,\left(x_{k}\right.$, $\left.y_{k}\right) \in R^{N} \times R$ where, $x_{i} \in R^{N}$ is an input parameter, 
$x_{i} \in R$ is the corresponding output parameter, and $k$ is the number of samples. Regression function can be expressed by the following formula:

$$
f(x)=\omega \cdot \theta(x)+b,
$$

where $b$ is the threshold and $\omega$ is the weight vector.

According to the theory of statistics, we define SVM regression function based on the target of minimization and build the following goal programming:

$$
\begin{aligned}
& R(\omega)=\min \left[\frac{1}{2}\|\omega\|^{2}+C \sum_{i=1}^{n}\left(\xi_{i}+\xi_{i}^{*}\right)\right] \\
& \text { s.t. } \quad y_{i}-f\left(x_{i}\right) \leqslant \varepsilon+\xi_{i} \\
& f\left(x_{i}\right)-y_{i} \leqslant \varepsilon+\xi_{i}^{*} \\
& \xi_{i}, \xi_{i}^{*} \geqslant 0
\end{aligned}
$$

where $\xi_{i}$ and $\xi_{i}^{*}$ are nonnegative slack variables; $C$ is the penalty factor, which indicates the degree of punishment for out of error samples and also a compromise between empirical risk and the complexity of the model; $\varepsilon$ is an insensitive loss function parameter.

By using Lagrange method to solve the constrained optimization problems described above, the original problem can be converted to its dual problem:

$$
\begin{aligned}
W\left(a_{i}, a_{i}^{*}\right)=\max & {\left[\frac{1}{2} \sum_{i=1}^{n} \sum_{j=1}^{n}\left(a_{i}-a_{i}^{*}\right)\left(a_{j}-a_{j}^{*}\right) K\left(x_{i}, x_{j}\right)\right.} \\
& \left.+\sum_{i=1}^{n} a_{i}^{*}\left(y_{i}-\varepsilon\right)-\sum_{i=1}^{n} a_{i}\left(y_{i}-\varepsilon\right)\right] \\
\text { s.t. } \quad & \sum_{i=1}^{n}\left(a_{i}-a_{i}^{*}\right) \\
& 0 \leqslant a_{i}, a_{i}^{*} \leqslant C,
\end{aligned}
$$

where $K\left(x_{i}, x_{j}\right)=\left[\theta\left(x_{i}\right) \cdot \theta\left(x_{j}\right)\right]$ is the kernel function of SVM and $a_{i}$ and $a_{i}^{*}$ are the Lagrange coefficients.

The SVM regression function is shown as follows:

$$
\begin{gathered}
f(x)=\sum_{i=1}^{n}\left(a_{i}-a_{i}^{*}\right) K\left(x_{i}, x_{j}\right)+b, \\
K\left(x_{i}, x_{j}\right)=\exp \left(-g\left|x_{i}-x_{j}\right|^{2}\right),
\end{gathered}
$$

where radial basis function (RBF) is used as the kernel function and $g$ is the width of the kernel function.

2.2.2. The Weighted Support Vector Machine Algorithm. The traditional support vector machine algorithm applies to the cases where the sample data obeys identically distribution and the samples are independent of each other, and it has same punishment for penalty parameters and error request parameters of different sample. In this case, the model can ensure an accuracy result. However, the actual data is more complex and we often find that some important samples demand lower training error while others allow a certain size of training error. Therefore, to get a more accurate regression estimate, we should assign different error requirements and penalty coefficients to each sample data when describing the optimization problem. To solve this problem, the weighted support vector machine regression algorithm [29] will be used to predict in this work.

Assume that the variance of random error term has the following properties:

$$
\begin{gathered}
E\left(\varepsilon_{i}\right)=0, \quad i=1, \ldots, l, \\
\operatorname{cov}\left(\varepsilon_{i}, \varepsilon_{j}\right)= \begin{cases}\sigma_{i}^{2}, & i=j \\
0, & i \neq j .\end{cases}
\end{gathered}
$$

In circumstances of different $\sigma_{i}^{2}$, it can be difficult for the traditional support vector machine regression model to achieve an ideal result. In the process of optimization, the status of each parameter $\xi$ and $\xi^{*}$ in the model is the same, which makes it easy for the regression line to be pulled to the larger variance items while the smaller variance items have relatively poor fitting degree. In this paper, to control the effects caused by differences in error term, we will adjust the impact of items on the summary of model by introducing the weight $\lambda_{i}$. The optimization model is

$$
\begin{gathered}
\min \frac{1}{2}\|\omega\|^{2}+C \sum_{i=1}^{l} \lambda_{i}\left(\xi_{i}+\xi_{i}^{*}\right), \\
\omega \cdot \phi(x)+b-y_{i} \leq \xi_{i}^{*}+\varepsilon t_{i}, \\
y_{i}-\omega \cdot \phi(x)-b \leq \xi_{i}+\varepsilon t_{i}, \\
\xi_{i}, \xi_{i}^{*} \geq 0, \quad i=1, \ldots, l,
\end{gathered}
$$

where $\lambda_{i}$ and $t_{i}$ are the weighted coefficients of the $i$ th training sample for parameters $C$ and $\varepsilon$, respectively. Literature [30] has pointed out that the weighting for penalty parameter $C$ of samples can greatly improve system performance, but the improvement is not so significant in the case of weighting for error requirements parameter $\varepsilon$; therefore, only optimal weighting for $\lambda_{i}$ will be executed in this paper.

The basic idea of gray correlation degree is to determine the degree of association according to similarities between curves. Therefore, to determine the weights, this paper introduces gray correlation degree to calculate the similarities between samples. The gray correlation degree is calculated as follows:

$$
\begin{aligned}
& \gamma\left(x_{0}(k), x_{i}(k)\right) \\
& =\frac{\min _{i} \min _{k} \Delta_{0 i}(k)+\varphi \max _{i} \max _{k} \Delta_{0 i}(k)}{\Delta_{0 i}(k)+\varphi \max _{i} \max _{k} \Delta_{0 i}(k)}, \\
& \Delta_{0 i}(k)=\left|x_{0}(k)-x_{i}(k)\right|, \quad \varphi \in[0,1] .
\end{aligned}
$$


The gray correlation degree $\gamma\left(x_{0}, x_{i}\right)$ is

$$
\gamma\left(x_{0}, x_{i}\right)=\frac{1}{n} \sum_{k=1}^{n} \gamma\left(x_{0}(k), x_{i}(k)\right) \text {. }
$$

The weight $\lambda_{i}$ is defined as

$$
\lambda_{i}=\frac{1}{\sum_{j=1}^{a} \gamma_{j}\left|S_{j}(i)-S_{j}(0)\right|+\varepsilon},
$$

where $i=1, \ldots, n, j$ is the number of independent variables, $\varepsilon$ is a relatively small number, and $S_{j}(i)$ is the data after the normalization. In practical application, each weight may be selected according to the actual situation.

\section{Case Study and Results Analysis}

Among the numerous factors influencing the ice coating on the transmission line, meteorological conditions are the most important factor, including temperature, humidity, wind speed, wind direction and other external climate. A lot of scholars have studied the impact factors preliminary. Literature [3] summarizes the three necessary conditions for the formation of icing by reviewing the results of research of predecessors. Air relative humidity must be above $85 \%$; wind speed should be greater than $1 \mathrm{~m} / \mathrm{s}$; the temperature have to reach $0^{\circ} \mathrm{C}$ and below.

On this basis, a pole called "Fuwaixian" in Hunan province is as a case study to demonstrate the effectiveness of the proposed approach. The historical meteorological data and ice thickness data from January 10, 2008, to March 21, 2008, are taken as data base. The forecasting model is solved through Matlab on a single core of a 32-bit Lenovo workstation running on Windows7 with 2 dual-core 2.60 GHz CPU and 4.0 GB of RAM. We extract rules from the past information to forecast the ice thickness. The main factors considered here are average temperature, relative humidity, wind speed, choosing average temperature, relative humidity and wind speed of forecasting day, the ice thickness, temperature, and relative humidity of the day before as input factors of support vector machine to predict the ice thickness of the transmission line. The original data chart is shown in Figure 3.

Before training samples, we must screen and normalize the raw data, deleting the cases of small relative humidity, high temperature, and small wind speed which lead to ice thickness close to 0. Finally, we retain 289 groups of data, of which the first 200 groups are the training set and the latter 90 groups are a test set, to prove the validity of the model.

The formula of normalization is as follows:

$$
\bar{x}=\frac{x-x_{\min }}{x_{\max }-x_{\min }} .
$$

After normalization, the values of each variable are between $[0,1]$, which eliminates the influence of dimension.

As the climate factors have the characteristics of greater volatility and randomness, SVM can take comprehensive consideration of multiple factors of ice thickness and has

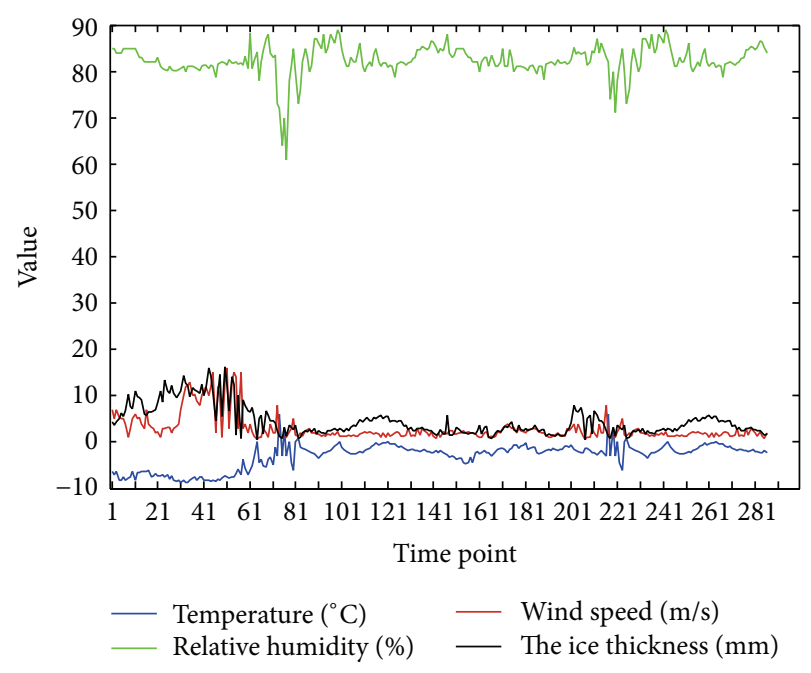

FIGURE 3: Original data chart of temperature, relative humidity, wind's speed, and ice thickness.

better ability of nonlinear mapping and generalization. As the selection of kernel parameter $g$ and penalty factor $C$ have great influence on the accuracy of the model of SVM regression, this paper selects Gauss radial basis function as kernel function of support vector machine model; that is,

$$
K\left(x, x_{i}\right)=\exp \left\{-\frac{\left|x-x_{i}\right|^{2}}{\delta^{2}}\right\} .
$$

This paper will adopt PSO-ACO hybrid cluster intelligent optimization algorithm to optimize the parameters $g$ and $C$ to find an optimal parameters to improve the accuracy of prediction.

This paper selects the average percentage error of predictive value and the actual value as the objective function to search for the target of the minimum value of the objective function. The optimal solution of ant colony corresponding to the global minimum is the parameters $g$ and $C$ of SVM when the iteration terminates. We predict the icing thickness after putting the optimized parameter into the SVM regression model.

Figure 4 shows the process of optimization iterative convergence of PSO-ACO parameters; we can see that MAPE presents the ladder convergence to the stationary after 100 iterations, the dynamic adjustment of SVM parameters. In the process of evolution, the minimum MAPE presenting in the tenth iteration convergence is $2.53 \%$; the corresponding optimal kernel function $g$ and the penalty factor $C$ are 3.145 and 41.916. Putting the obtained parameters into the weighted SVM model, we get the prediction of ice thickness shown in Figure 5.

In order to assess the reasonableness of the model proposed in this paper, we select support vector machine optimized by ant colony (ACO-SVM), SVM model, and linear regression model as the model for comparison. The prediction results of the three algorithms are shown in Figure 6 and the error is shown in Figure 7. 


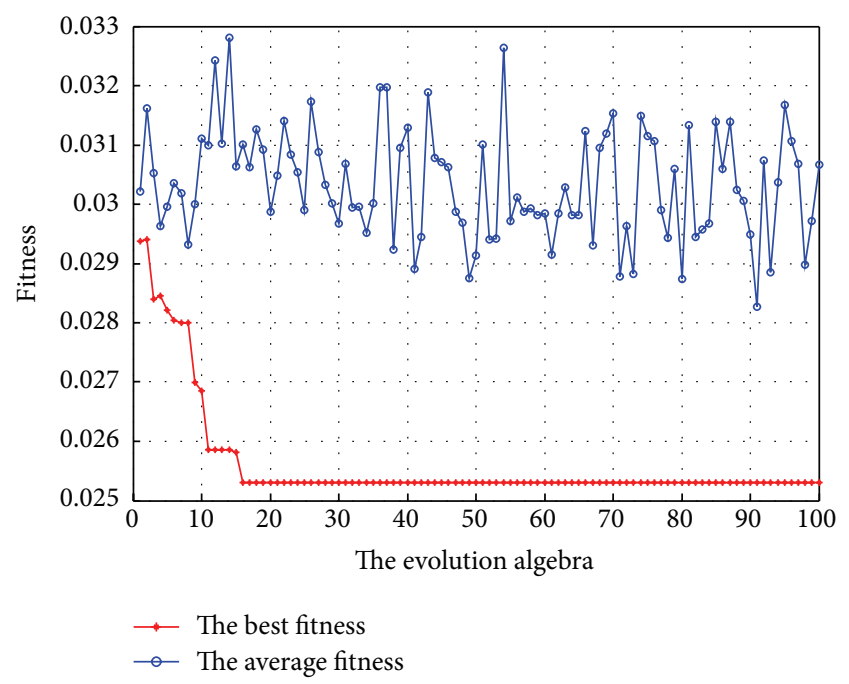

FIgURE 4: Process of optimization iterative convergence of PSOACO parameters.

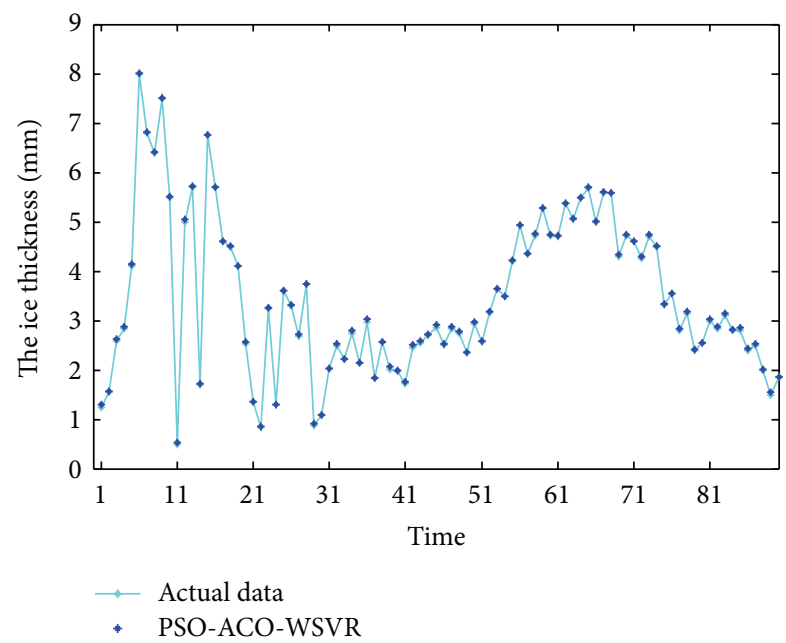

FIGURE 5: Forecasting curve of WSVR based on PSO-ACO hybrid optimization.

This paper selects the mean absolute percentage error index as quantitative evaluation of the result of prediction:

$$
\operatorname{MAPE}=\frac{1}{n} \sum_{i=1}^{n}\left|\frac{\widehat{Y}_{i}-Y_{i}}{Y_{i}}\right| .
$$

Error distributions of different methods are shown in Figure 7.

It can be seen from comparison of the prediction curve and the actual load curve that the results of four algorithms of ice thickness have approximation to actual curve. Among them, weighted support vector machine regression (WSVR) based on PSO-ACO hybrid intelligent optimization has the best fitting effect, and the mean absolute percentage error prediction result of this proposed method is $2.533 \%$ while ACOSVM, the traditional SVM and linear regression method are $6.47 \%, 10.62 \%, 12.24 \%$. The error is much higher than

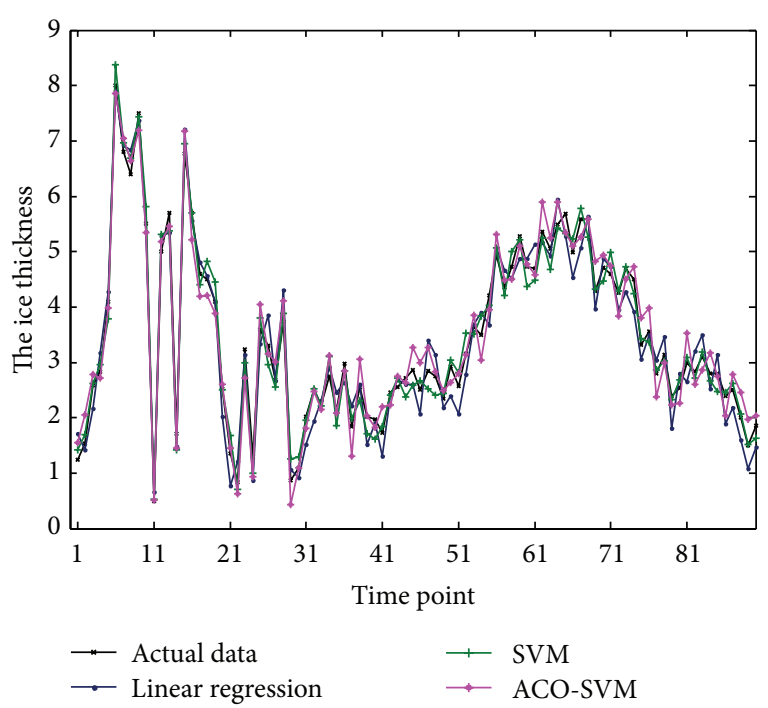

FIGURE 6: Comparison of the prediction results of different algorithms.

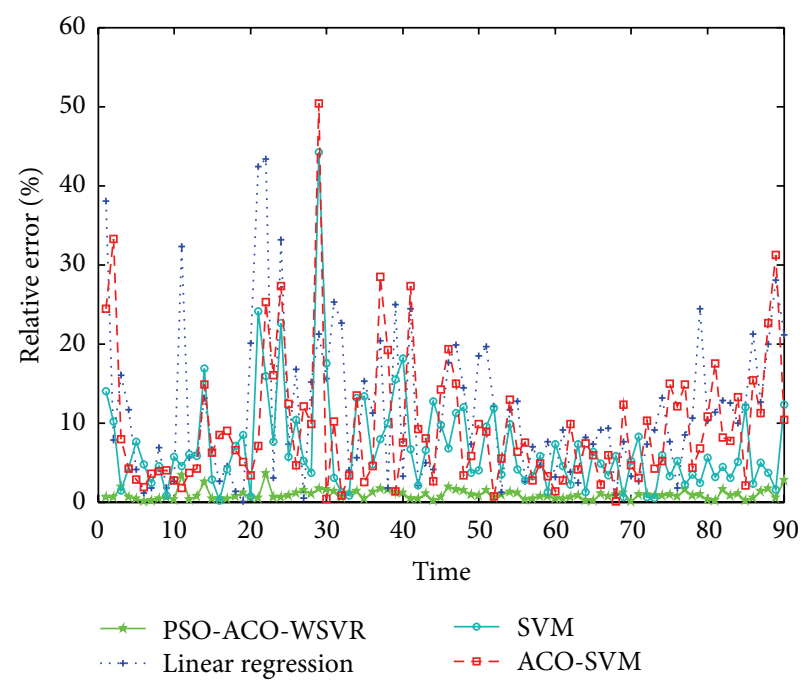

FIgURE 7: The error distribution of different algorithms.

the method proposed in this paper. Error evaluation results show that weighted support vector machine optimized by the hybrid algorithm has a higher prediction accuracy than the single optimization SVM algorithm, the hybrid optimization algorithm makes up for the defects of single algorithm, and weighted support vector machine makes full use of the sample information, which improve the accuracy and the generalization ability of the model and make better prediction effect. Compared with other methods, the proposed method has obvious advantages and can be used for the ice thickness prediction of transmission line.

\section{Conclusions}

This paper proposes an intelligent model of weighted support vector machine (WSVR) based on PSO-ACO hybrid 
optimization. It is used to predict the ice thickness of transmission line, which has extremely important realistic meaning for the power sector to control ice coating effectively. This intelligent model is combined with the advantages of dealing with small samples, nonlinearity, and local optimum of SVM. The PSO-ACO hybrid optimization algorithm can overcome shortcomings of the local optimum in particle swarm algorithm and lack of pheromone in ant colony algorithm; at the same time, weighted support vector machine can make full use of sample information, which improves the generalization ability of the mode and promote the actual application scope of the prediction model. During the process of thickness forecasting, we give full consideration of the impact factors of climate and select the key factors, which makes the model more credible and scientific. This paper selects the icing data of "Fuwaixian" tower in a certain region of Hunan province from January 10, 2008, to March 21, 2008, for calculation. Empirical results show that the accuracy and generalization ability of improved model are improved.

\section{Conflict of Interests}

The authors declare that there is no conflict of interests regarding the publication of this paper.

\section{Acknowledgments}

This work was supported by Natural Science Foundation of China (Project nos. 71471059 and 7107152). The authors would like to thank the anonymous reviewers for their valuable comments, which greatly helped them to clarify and improve the contents of the paper.

\section{References}

[1] C. G. Li, Y. Z. Lv, and X. Cui, "The problem of safe operation of power grid in China under conditions of ice and snow disasters," Power System Technology, vol. 32, no. 4, pp. 14-21, 2008.

[2] L. L. Shi, "Assessment of forest damage caused by ice storm based on MODIS data-a case study of Jiangxi Province, China," Disaster Advances, vol. 6, no. 7, pp. 67-72, 2013.

[3] H. Z. Liu, DC Transmission Line Icing and Control, China Electric Power Press, Beijing, China, 2012.

[4] J. Z. Lu, Z. L. Jiang, H. C. Lei et al., "Analysis of Hunan power grid ice disaster accident in 2008," Dianli Xitong Zidonghua/Automation of Electric Power Systems, vol. 32, no. 11, pp. 16-19, 2008.

[5] I. Imai, "Studies on ice accretion," Researches on Snow and Ice, vol. 3, no. 1, pp. 35-34, 1953.

[6] P. M. Chaine and G. Casfonguay, "New approach to radial ice thichness concept applied to bundle-like conductors," Industial Metecrology Study, Environment Canada, Toronto, Canada, 1974.

[7] P. McComber, J. de Lafontaine, J. Druez et al., "A comparison of neural network and multiple regression transmission Line Icing models," in Proceedings of the 55th Eastern Snow Conference, June 1998.

[8] P. Li, N. Li, and M. M. Cao, "Micro-meteorology features extraction and status assessment for transmission line icing based on intelligent algorithms," Journal of Information \& Computational Science, vol. 7, no. 10, pp. 2043-2052, 2010.

[9] J. Liu, A. J. Li, and L. P. Zhao, "Prediction model based on fuzzy and T-S neural network for ice thickness," Hunan Electric Power, vol. 32, no. 3, pp. 1-4, 2012.

[10] X. N. Huang and J. H. Xu, "Prediction of transmission line icing based on data driven algorithm and LS-SVM model," Automation of Electric Power Systems, vol. 38, no. 15, pp. 81-86, 2014.

[11] D. Liu, D. Niu, H. Wang, and L. Fan, "Short-term wind speed forecasting using wavelet transform and support vector machines optimized by genetic algorithm," Renewable Energy, vol. 62 , pp. 592-597, 2014.

[12] D. Dai and X. T. Huang, "Prediction of transmission line icing based on support vector machine model," High Voltage Technology, vol. 39, no. 11, pp. 2822-2828, 2013.

[13] Z. Xiang and C. Lin, "Support vector machine fault diagnosis based on optimization algorithm of Drosophila melanogaster," Electronic Design Engineering, vol. 16, pp. 90-93, 2013.

[14] Y. F. Liao and L. J. Duan, "Estimation of icing thickness on Hunan wire," Journal of Atmospheric Sciences, vol. 33, no. 4, pp. 395-400, 2010.

[15] X. Wu, P. J. Sun, and H. X. Xiong, "Establishment of icing model using conventional meteorological data," Journal of Atmospheric Sciences, no. 3, pp. 335-341, 2012.

[16] F. Kaytez, M. C. Taplamacioglu, E. Cam, and F. Hardalac, "Forecasting electricity consumption: a comparison of regression analysis, neural networks and least squares support vector machines,' International Journal of Electrical Power \& Energy Systems, vol. 67, pp. 431-438, 2015.

[17] M. Farzaneh and K. Savadjiev, "Statistical analysis of field data for precipitation icing accretion on overhead power lines," IEEE Transactions on Power Delivery, vol. 20, no. 2, pp. 1080-1087, 2005.

[18] J. C. Liu and H. T. Wang, "Forecast model for inner corrosion rate of oil pipeline based on GA-SVM," Information, vol. 15, no. 11, pp. 4725-4730, 2012.

[19] Q. Zhang, K. K. Lai, D. Niu, Q. Wang, and X. Zhang, "A fuzzy group forecasting model based on least squares support vector machine (LS-SVM) for short-term wind power," Energies, vol. 5, no. 9, pp. 3329-3346, 2012.

[20] L. Chen, Research on the Prediction of Short Term Electricity Load Based on Weighted Similarity and Weighted Support Vector Machine, South China University of Technology, 2012.

[21] T. X. Wei, G. W. Ma, and W. B. Huang, "Prediction of runoff based on penalized weighted support vector machine regression model," Journal of Hydroelectric Engineering, vol. 31, no. 6, pp. 35-38, 2012.

[22] J. Liang and D. Wu, "Smooth diagonal weighted Newton support vector machine," Mathematical Problems in Engineering, vol. 2013, Article ID 349120, 10 pages, 2013.

[23] Q. Ye, M. Wang, and J. R. Han, "Integrated risk governance in the Yungui Plateau, China: the 2008 icesnow storm disaster," Journal of Alpine Research, vol. 100, no. 4, pp. 100-112, 2012.

[24] S. W. Yao, Research on Target Tracking Direction Based on Swarm Intelligence and Particle Filter Algorithm, China University of Geosciences, 2012.

[25] D. X. Niu, L. Zhao, B. Zhang, and H. F. Wang, "Application of particle swarm grey model in power load forecasting," Chinese Journal of Management Science, vol. 15, no. 1, pp. 69-73, 2007. 
[26] D. X. Niu, J. C. Li, J. Y. Li, and D. Liu, "Middle-long power load forecasting based on particle swarm optimization," Computers and Mathematics with Applications, vol. 57, no. 11-12, pp. 18831889, 2009.

[27] W. C. Zhang, Application of Ant Colony and Particle Swarm Hybrid Optimization Algorithm, Tianjin University, 2007.

[28] J. Wang, L. Li, D. X. Niu, and Z. Tan, "An annual load forecasting model based on support vector regression with differential evolution algorithm," Applied Energy, vol. 94, pp. 65-70, 2012.

[29] S. Bang and M. Jhun, "Weighted support vector machine using $k$-means clustering," Communications in Statistics-Simulation and Computation, vol. 43, no. 10, pp. 2307-2324, 2014.

[30] F. E. H. Tay and L. Cao, "Application of support vector machines in financial time series forecasting," Omega, vol. 29, no. 4, pp. 309-317, 2001. 


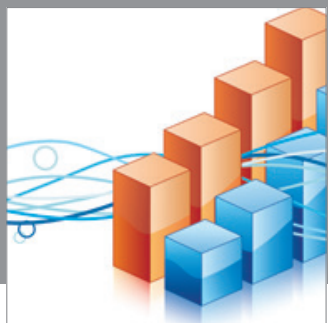

Advances in

Operations Research

mansans

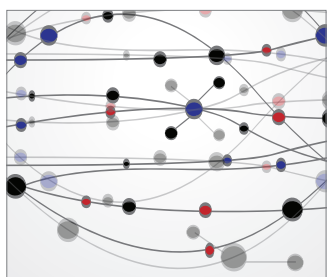

The Scientific World Journal
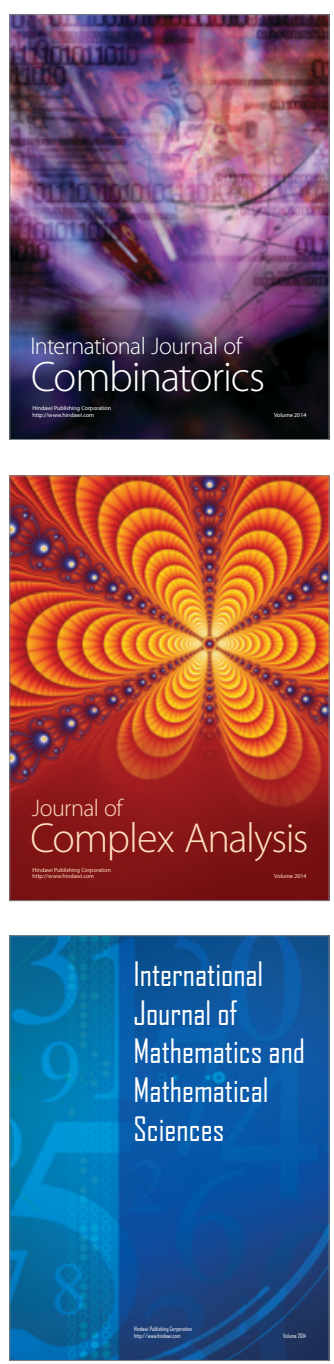
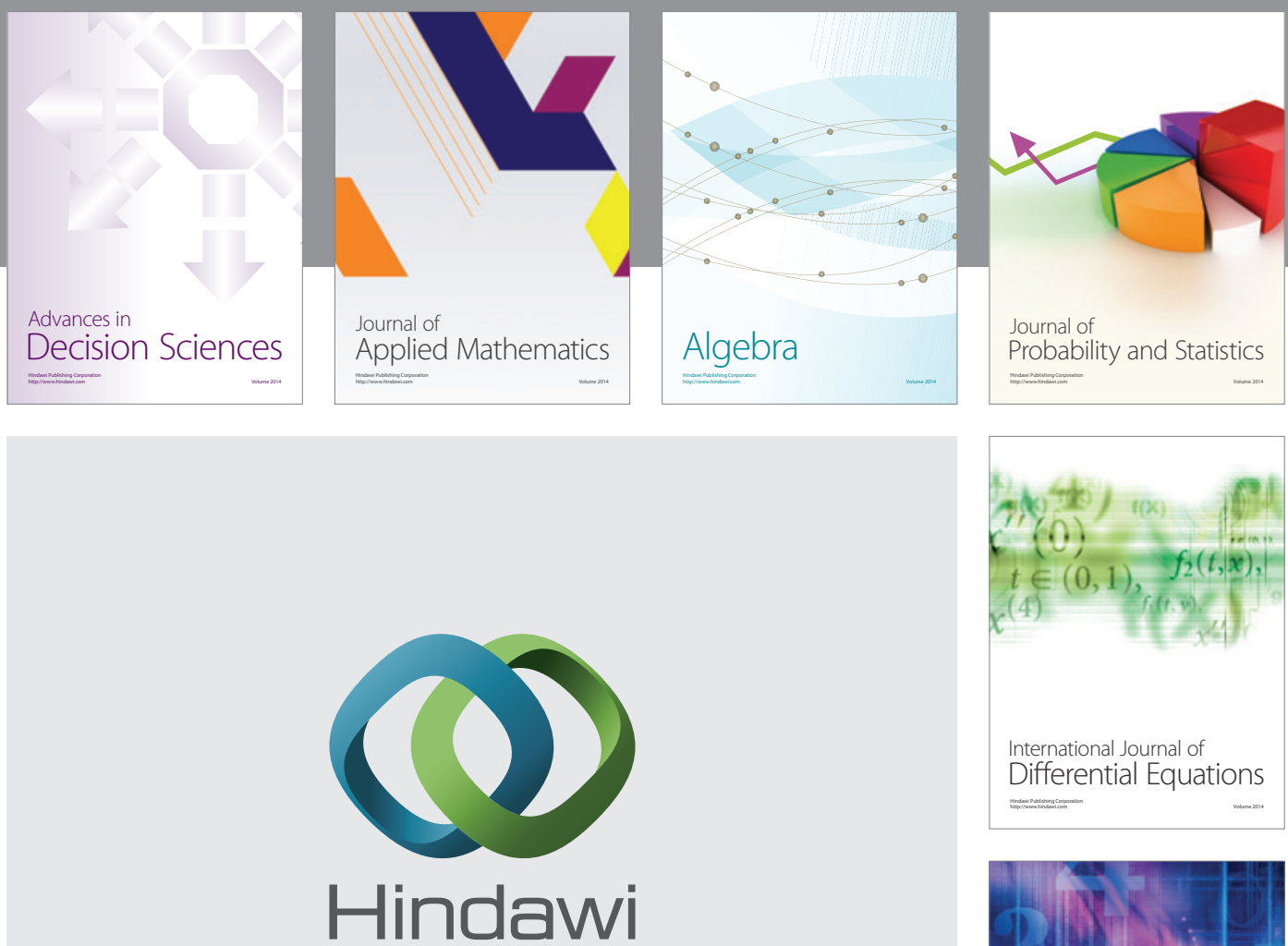

Submit your manuscripts at http://www.hindawi.com
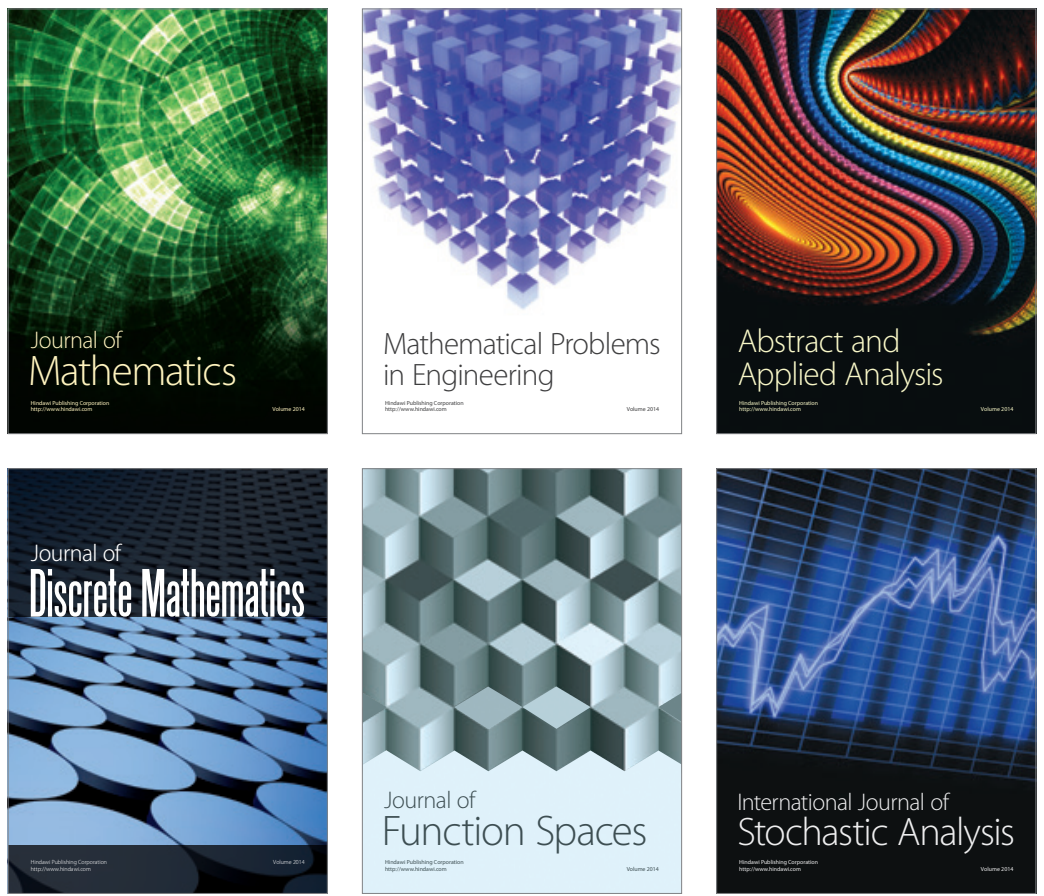

Journal of

Function Spaces

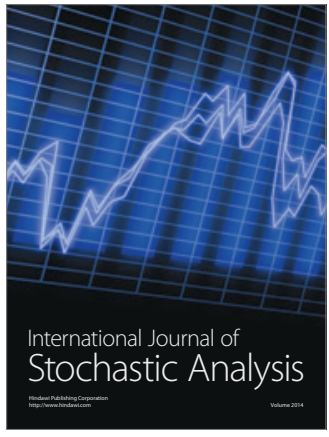

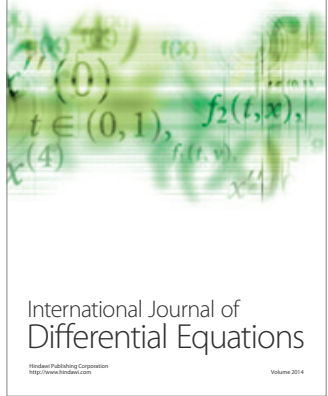
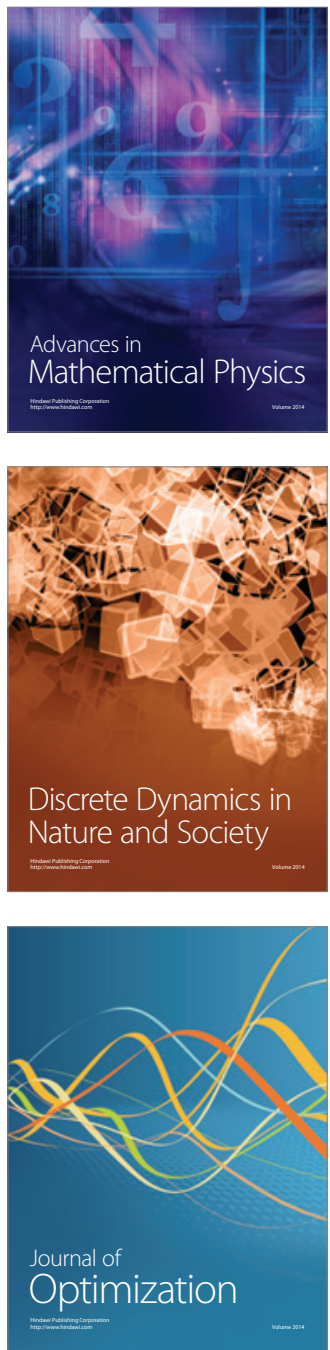